\title{
Triggered massive-star formation on the borders of Galactic H II regions
}

\section{Star formation at the periphery of Sh2-212 ${ }^{\star}, \star \star$}

\author{
L. Deharveng ${ }^{1}$, B. Lefloch ${ }^{2}$, S. Kurtz ${ }^{3}$, D. Nadeau ${ }^{4}$, M. Pomarès ${ }^{1}$, J. Caplan ${ }^{1}$, and A. Zavagno ${ }^{1}$ \\ 1 Laboratoire d'Astrophysique de Marseille, 2 place Le Verrier, 13248 Marseille Cedex 4, France \\ e-mail: lise.deharveng@oamp.fr \\ 2 Laboratoire d'Astrophysique de l'Observatoire de Grenoble, 414 rue de la Piscine, BP 53, 38041 Grenoble Cedex 9, France \\ 3 Centro de Radioastronomía y Astrofísica, UNAM, Apartado Postal 3-72, 58089, Morelia, Michoacán, México \\ ${ }^{4}$ Observatoire du Mont Mégantic et Département de Physique, Université de Montréal, CP 6128, Succ. Centre-Ville, Montréal, \\ H3C3J7, QC, Canada
}

Received 11 December 2007 / Accepted 8 February 2008

\section{ABSTRACT}

\begin{abstract}
Aims. We wish to establish whether sequential star formation is taking place at the periphery of the Galactic H II region Sh2-212. Methods. We present CO millimetre observations of this region obtained at the IRAM 30-m telescope to investigate the distribution of associated molecular material. We also use deep JHK observations obtained at the CFHT to study the stellar content of the region, and radio observations obtained at the VLA to look for the presence of an ultra-compact (UC) H II region and for maser emission. Results. In the optical, Sh2-212 is spherically symmetric around its central exciting cluster. This H II region is located along a molecular filament. A thin, well-defined half ring of molecular material surrounds the brightest part of the $\mathrm{H}$ II region at the rear and is fragmented. The most massive fragment $\left(\sim 200 M_{\odot}\right)$ contains a massive young stellar object displaying a near-IR excess; its spectral energy distribution indicates a high-mass $\left(\sim 14 M_{\odot}\right)$, high-temperature $(\sim 30000 \mathrm{~K})$, and high-luminosity $\left(\sim 17000 L_{\odot}\right)$ source. This object ionizes a UC H II region.

Conclusions. Sh2-212 is a good example of massive-star formation triggered via the collect and collapse process. The massive YSO observed at its periphery is a good candidate for a massive star formed in isolation.
\end{abstract}

Key words. stars: formation - stars: early-type - ISM: H II regions - ISM: individual objects: Sh2-212

\section{Introduction}

Various mechanisms may trigger star formation on the borders of $\mathrm{H}$ II regions (see the review by Elmegreen 1998). All rely on the high-pressure exerted by the warm ionized gas on the surrounding cold neutral material. These mechanisms differ in their assumptions concerning the nature of the surrounding medium (homogeneous or not) and the part played by turbulence. One of these mechanisms, the collect and collapse process, first proposed by Elmegreen \& Lada (1977), is particularly interesting as it allows the formation of massive fragments (hence subsequently of massive objects, stars or clusters), out of an initially uniform medium. In this process a layer of neutral material is collected between the ionization front (IF) and the associated shock front (SF) during the supersonic expansion of an $\mathrm{H}$ II region. With time this layer may become massive and gravitationally instable, leading to the formation of dense massive cores (Whitworth et al. 1994; Hosokawa \& Inutsuka 2006).

\footnotetext{
* Based on observations obtained at the IRAM, Spain, at the CFHT, Hawaii, at the VLA, USA, and at the Observatoire de Haute-Provence, France.

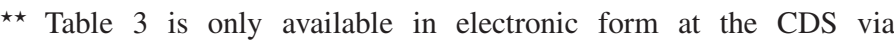
anonymous ftp to cdsarc.u-strasbg.fr (130.79.128.5) or via http://cdsweb.u-strasbg.fr/cgi-bin/qcat?]/A+A/482/585
}

We have previously proposed seventeen Galactic H II regions as candidates for the collect and collapse process of massivestar formation (Deharveng et al. 2005). Among these is Sh2212, the subject of the present paper, described in Sect. 2. Our main criterion for the choice of Sh2-212 was the presence of a bright MSX point source (Price et al. 2001) at its periphery, beyond the ionization front, coincident with a red object in the 2MASS survey (Skrutskie et al. 2006) and with a small optical reflection nebula. However nothing was yet known about Sh2-212's molecular environment. We now present new highresolution molecular observations to investigate the distribution of molecular material. Do we observe a layer of dense neutral material surrounding the ionized gas - a signature of the collect and collapse process of star formation? These observations are described in Sect. 4. We also present new JHK observations to determine the stellar content of this region. Are young stellar objects (YSOs) present on the border of Sh2-212? What is the nature of the MSX point source observed near the IF? This is discussed in Sect. 3. We also present new radio observations aimed at detecting possible UC H II regions at the periphery of Sh2-212, and at detecting maser emission indicative of recent star formation. These observations are described in Sect. 5.

The results are discussed in Sect. 6, where we present our view of the morphology of the whole complex, and argue in 


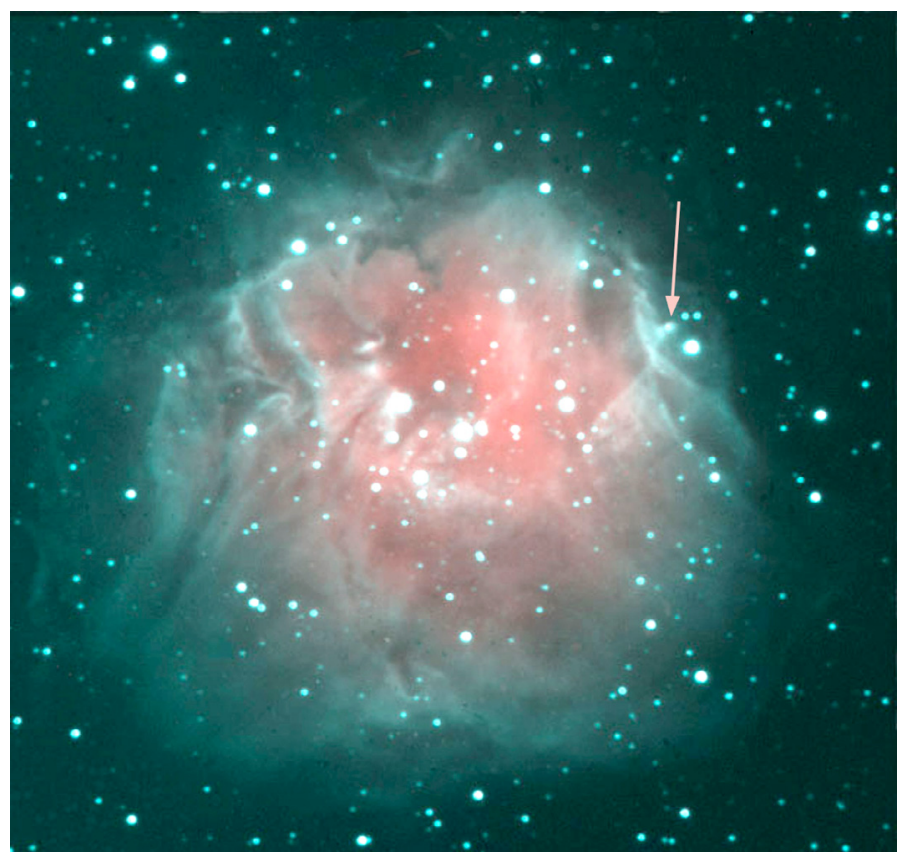

Fig. 1. Composite colour image of Sh2-212 in the optical. North is up and east is left. The size of the field is 7!.0 (E-W) $\times 6{ }^{\prime} 6(\mathrm{~N}-\mathrm{S})$. Pink corresponds to the $\mathrm{H} \alpha 6563 \AA$ emission, and turquoise to the [S II] $6717 \AA+6731 \AA$ emission, enhanced near the ionization front. The arrow points to the reflection nebulosity, associated with star No. 228 , discussed in the text.

favour of the collect and collapse process of massive-star formation.

\section{Description of the region}

Sh2-212 (Sharpless 1959) is a bright optically-visible H II region in the outer Galaxy $\left(l=155^{\circ} .36, b=2^{\circ} .61\right)$. It lies high above the Galactic plane $(\sim 300 \mathrm{pc}$ assuming a distance of $6.5 \mathrm{kpc}$, Sect. 2.1), and far from the Galactic centre $(14.7 \mathrm{kpc})$. Its diameter is $\sim 5^{\prime}(9.5 \mathrm{pc})$. It is a high-excitation $\mathrm{H}$ II region, ionized by a cluster containing an O5.5neb (Moffat et al. 1979) or an O6I star (Chini \& Wink 1984).

Figure 1 presents a colour image of Sh2-212 in the optical, a composite of two frames obtained at the $120-\mathrm{cm}$ telescope of the Observatoire de Haute-Provence. Pink corresponds to the $\mathrm{H} \alpha$ emission at $6563 \AA$ (exposure time $1 \mathrm{~h}$ ) and turquoise to the [S II] emission at $6717 \AA$ and $6731 \AA$ (exposure time $2 \times 1 \mathrm{~h}$ ). [S II] is enhanced near the ionization front, and thus is a good tracer of the limits of the ionized region and of the morphology of the ionization front. Sh2-212 appears as a circular $\mathrm{H}$ II region around its exciting cluster. Numerous substructures are present, indicating that Sh2-212 is presently evolving in a non-homogeneous medium. A bright rim is conspicuous at the north-western border of Sh2-212. A small reflection nebula (indicated by an arrow in Fig. 1) is present beyond this ionization front.

Because Sh2-212 is both optically bright and situated far $(14.7 \mathrm{kpc})$ from the Galactic centre, it has been included in numerous studies of abundance determinations in the Galaxy. For this purpose absolute integrated line fluxes in a number of nebular emission lines were measured through a circular diaphragm by Caplan et al. (2000). These measurements confirm the high excitation of Sh2-212; they indicate an electron temperature
Table 1. Coordinates of the objects discussed in the text.

\begin{tabular}{lllllll}
\hline \hline Object & \multicolumn{3}{c}{ RA (2000) } & \multicolumn{3}{c}{ Dec (2000) } \\
& $\mathrm{h}$ & $\mathrm{m}$ & $\mathrm{s}$ & $\circ$ &, & " \\
\hline Main exciting star M2 & 4 & 40 & 37.44 & +50 & 27 & 40.5 \\
MSX G155.3319+02.5989 & 4 & 40 & 27.2 & +50 & 28 & 29 \\
IRAS 04366+5022 & 4 & 40 & 26.1 & +50 & 28 & 24 \\
UC H II region & 4 & 40 & 27.2 & +50 & 28 & 29 \\
Massive YSO No. 228 & 4 & 40 & 27.24 & +50 & 28 & 29.5 \\
\hline
\end{tabular}

Table 2. Velocity measurements.

\begin{tabular}{lll}
\hline \hline Line & $V_{\text {LSR }}\left(\mathrm{km} \mathrm{s}^{-1}\right)$ & Reference \\
\hline $\mathrm{H} \alpha$ & -39.5 & Pişmiş et al. (1991) \\
$\mathrm{H} \alpha$ & $-43.9 \pm 0.1$ & Fich et al. (1990) \\
$\mathrm{H} 109 \alpha$ & $-40.1 \pm 1.0$ & Lockman (1989) \\
$\mathrm{CO}$ & $-35.3 \pm 0.3$ & Blitz et al. (1982) \\
$\mathrm{CO}$ & -34.3 & Shepherd \& Churchwell (1996) \\
\hline
\end{tabular}

of $9700 \mathrm{~K}$ and an electron density of $130 \mathrm{~cm}^{-3}$ (Deharveng et al. 2000).

The coordinates of the objects discussed in the text are given in Table 1.

Sh2-212 is a thermal radio-continuum source, with a flux density of $1.58 \mathrm{Jy}$ at $1.46 \mathrm{GHz}$ (Fich 1993, and references therein). The angular resolution of Fich's observations, 40", was insufficient for the detection of a possible UC H II region on the border of Sh2-212. Higher angular resolution radio observations will be presented and discussed in Sect. 5 .

Table 2 lists the velocities, obtained by various authors, of the ionized gas and the associated molecular material. On the whole, the ionized gas flows away from the molecular cloud, with a radial velocity of the order of $5 \mathrm{~km} \mathrm{~s}^{-1}$. This may be indicative of a "champagne flow" (Tenorio-Tagle 1979). This point will be developed in Sect. 6.

Sh2-212 was proposed by Deharveng et al. (2005) as a candidate for the collect and collapse process of massive-star formation, on the basis of $i$ ) the presence of a ring of MSX emission at $8.3 \mu \mathrm{m}$ (mainly PAH emission) surrounding the brightest part of the ionized region; this indicates the presence of dense neutral material and dust around the ionized gas; and ii) the presence of a luminous MSX point source in the direction of this dust ring (indicated by an arrow in Fig. 2). This MSX point source lies in the direction of the reflection nebulosity. Figure 2 shows that the bright ring of MSX emission at $8.3 \mu \mathrm{m}$ surrounds only the bright northern part of the ionized region, and not the whole region. However, fainter brightness emission is observed around the southern part of the H II region. This point will be discussed in Sect. 6.

\subsection{The distance of Sh2-212}

A kinematical distance $D$ can be estimated from the velocity of the molecular gas, $V_{\mathrm{LSR}}(\mathrm{CO})=-35 \pm 1 \mathrm{~km} \mathrm{~s}^{-1}$ (Table 2), and from the Galactic rotation curve of Brand \& Blitz (1993); we obtain $D=6.1 \pm 0.4 \mathrm{kpc}$.

A photometric distance can be estimated for M2, the main exciting star of Sh2-212. We have used the spectral type and the $U B V$ magnitudes of Moffat et al. (1979; O5.5V, $U=11.90$, $B=12.34, V=11.77$ ), our $J H K$ magnitudes (Sect. $4 ; J=10.38$, $H=10.17, K=10.07)$, the synthetic photometry of $\mathrm{O}$ stars 


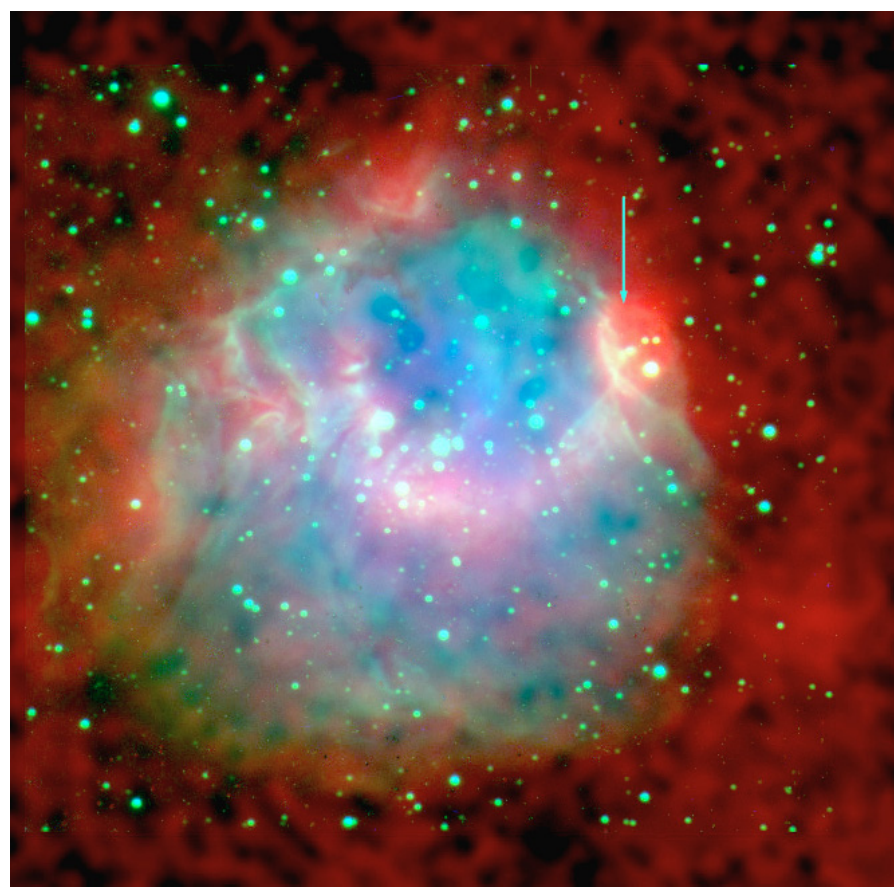

Fig. 2. Composite colour image of Sh2-212 in the optical and the midIR. Red corresponds to the MSX emission at $8.3 \mu \mathrm{m}$, and turquoise to the optical emission of the ionized gas. The arrow points to the MSX point source associated with star No. 228 and the UC H II region discussed in the text.

by Martins \& Plez (2006), and the interstellar extinction law of Rieke \& Lebofsky (1985). The best fit is obtained for a distance of $6.5 \mathrm{kpc}$ and a visual extinction $A_{V}$ of $2.85 \mathrm{mag}$.

In the following we adopt this distance of $6.5 \mathrm{kpc}$, which is consistent with the kinematic one.

\section{Near IR observations}

\subsection{Observations}

Sh2-212 was observed with the CFHT-IR camera on the night of 2002 October 20. Frames were obtained in the $J H K$ broadband filters. The detector was a Rockwell array of $1024 \times 1024$ pixels, with a pixel size of 0.211 . For each band a mosaic of nine positions was obtained, each position being observed ten times with a short exposure time. This results in a total field of view of $4.4(\mathrm{E}-\mathrm{W}) \times 5.2(\mathrm{~N}-\mathrm{S})$, and total integration times of $270 \mathrm{~s}$, $270 \mathrm{~s}$, and $450 \mathrm{~s}$ in the $J, H$, and $K$ bands respectively.

The $J, H$, and $K$ images were reduced using the DAOPHOT stellar photometry package (Stetson 1987), with PSF fitting. The results were calibrated using the 2MASS Point Source Catalog (Skrutskie et al. 2006), with 65 common stars. After a bestfit transformation, an rms dispersion of $0.10 \mathrm{mag}$ is present between our photometry and 2MASS, in each band and each colour. A total of 891 sources were measured in the three $J H K$ bands, and 36 more were measured in only one or two bands. The detection limit is $\sim 17.5 \mathrm{mag}$ in $J$ and $K$, and $\sim 18 \mathrm{mag}$ in $H$. The seeing was 0.9 .

Figure 3 presents a composite $J H K$ colour image of Sh2212. Our observations do not cover the whole H II region (our field is centred on the massive YSO, star No. 228, discussed below). We have supplemented the coverage of the Sh2-212 field, when necessary, using the 2MASS survey. Table 3, giving the

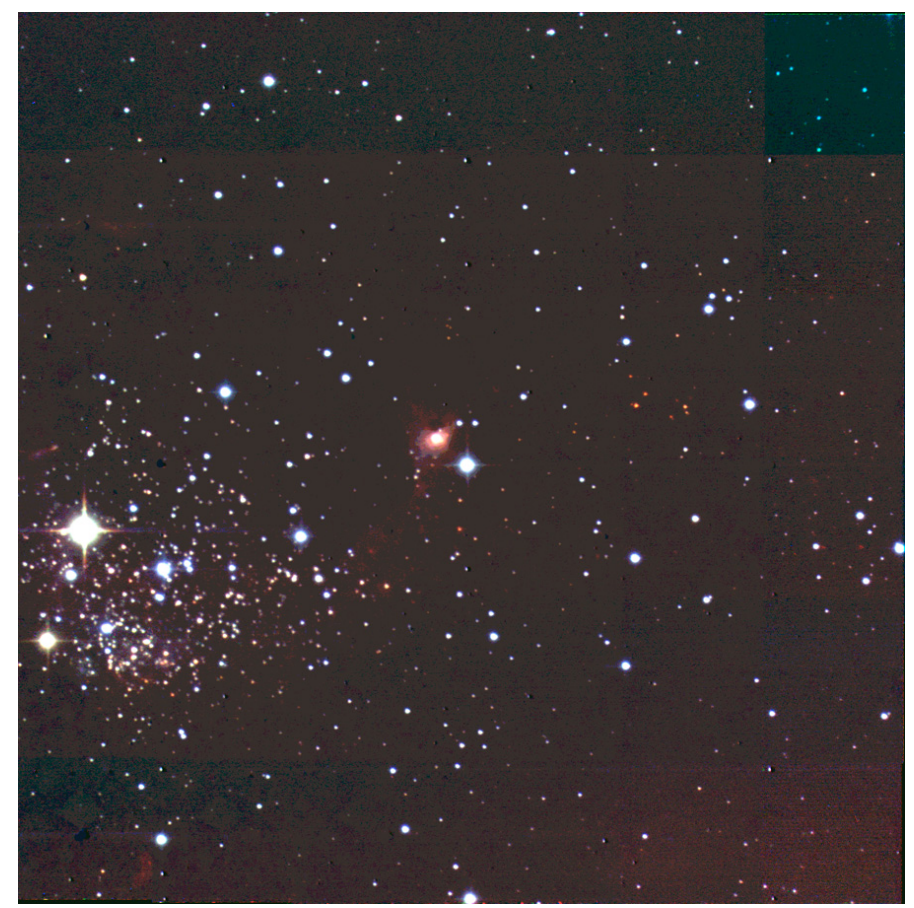

Fig. 3. Sh2-212. Composite colour image of Sh2-212 in the near-IR ( $J$ is blue, $H$ is green, $K$ is red). The colours of the stars are mainly determined by extinction. North is up and east is left. The size of the field is $4.4(\mathrm{E}-\mathrm{W}) \times 5 ! 2(\mathrm{~N}-\mathrm{S})$. The red nebulosity at the centre of the field corresponds to the optical reflection nebula associated with the MSX point source and star No. 228.

coordinates and the magnitudes of the stars in the $J H K$ bands (CFHT observations), is available in electronic form at the CDS.

\subsection{The stellar population associated with Sh2-212}

Figure 4 presents the $K$ versus $J-K$ magnitude-colour diagram of the sources detected in the three bands. The main sequence is drawn for a distance of $6.5 \mathrm{kpc}$, using the absolute calibration and colours of Martins et al. (2005) and Martins \& Plez (2006) for $\mathrm{O} 3$ to $\mathrm{O} 9.5$ stars; for later spectral types the absolute calibration is that of Schmidt-Kaler (1982) and the colours are from Tokunaga (2000). Note that there is some overlap between the magnitudes of $\mathrm{O} 9.5$ and B0 stars. The reddening lines correspond to a visual extinction of $30 \mathrm{mag}$. The interstellar extinction law is from Indebetouw et al. (2005).

The $J-H$ versus $H-K$ colour-colour diagram is presented in Fig. 5. The reddening lines are drawn for a visual extinction of 20 mag. They bracket the region occupied by reddened mainsequence stars. Stars near or above the upper reddening line may be evolved stars (giants). Stars below the bottom reddening line have a near-IR excess; they are probably young stellar objects associated with large amounts of dust (in an envelope or a disk), such as T Tauri stars, Herbig Ae/Be stars and more massive YSOs (Lada \& Adams 1992).

We have labelled a few objects in Fig. 6:

the stars observed in $U B V$ by Moffat et al. (1979), each marked with the letter $M$ followed by the number given by these authors.

the stellar object observed in the direction of the MSX and IRAS point sources. The large symbols in Figs. 4 and 5 correspond to the magnitudes and colours of the whole object (star No. 228 plus associated nebulosity) 
Table 3. Coordinates and $J H K$ photometry of all the stars in a 4.4 $\times 5.2$ field centred on star No. 228. This table is only available in electronic form at the CDS.

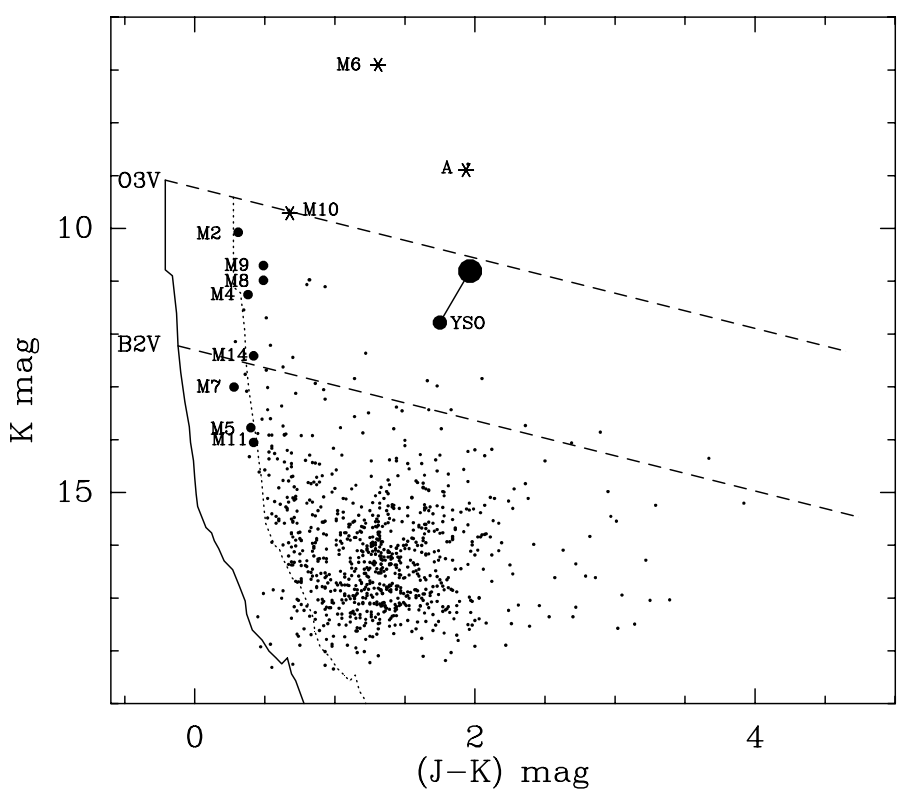

Fig. 4. $K$ versus $J-K$ diagram. The main sequence is drawn for a visual extinction of zero (full line) and $3 \mathrm{mag}$ (dotted line). The reddening lines, corresponding to a visual extinction of $30 \mathrm{mag}$, are issued from $\mathrm{O} 3 \mathrm{~V}$ and B2V stars. A few stars are identified, according to Fig. 6. Moffat et al.'s (1979) stars are identified by their number according to these authors. The asterisks are for 2MASS measurements. The connected full circles are for the star No. 228 (the YSO) and its associated nebulosity.

integrated in a diaphragm of radius $66^{\prime \prime} 3$. Note that this object presents a near-IR colour excess.

- a few other stars, saturated in our frames, are identified by asterisks; their magnitudes are from the 2MASS catalogue.

Figure 4 shows that the whole region is affected by a visual extinction of about $3 \mathrm{mag}$, most probably of interstellar origin. Moffat et al.'s stars are, at optical wavelengths, the brightest components in the observed field; most of these stars follow the main sequence reddened by $\sim 3$ mag. In particular, this is the case of M2, an O5.5 star according to Moffat et al., and the main exciting star of Sh2-212. Our JHK photometry confirms this conclusion. However, Figs. 4 and 5 show that a few of Moffat et al.'s stars are evolved; this is the case for stars M 6, M 10, and possibly M 8 and M9. Star A, very bright in the near IR but not in the optical (not measured by Moffat et al.), is also an evolved star. (Fig. 4 shows that it is too luminous to be a main-sequence star associated with Sh2-212, and Fig. 5 shows it to be a giant star.) It is not possible to know whether these stars belong to the exciting cluster or if they are unrelated foreground stars. Our near-IR images also show that stars M 1 and M 3 are double stars.

Red stars are observed all over the field of Fig. 3. Their density is especially high in the direction or in the vicinity of the molecular condensation C2 (Sect. 4.2). Many of these objects present a near-IR excess, indicating that they are YSOs.

The extinction affecting the central cluster of Sh2-212 (about $3 \mathrm{mag}$ ) is very low for the distance of the region and is thus probably mainly of interstellar origin. Thus very little local dust is present in front of the optical nebula and its exciting cluster.

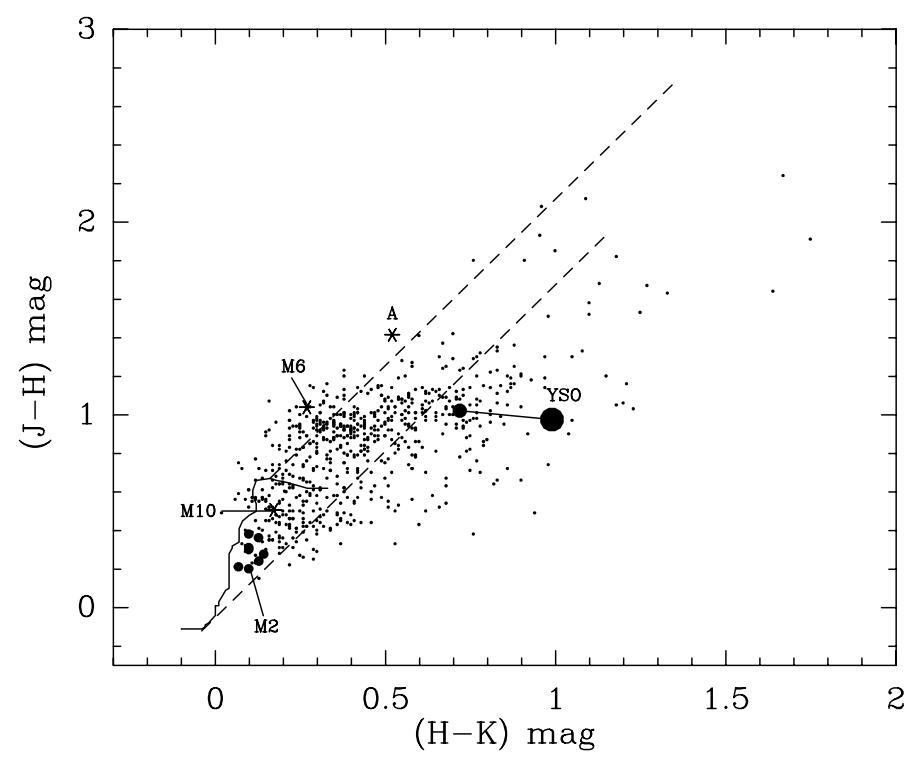

Fig. 5. $J-H$ versus $H-K$ diagram for stars with $K \leq 17$ mag. The main sequence (full line) is drawn for a visual extinction of zero. The reddening lines, starting at the positions of $\mathrm{B} 0 \mathrm{~V}$ and M0V stars, have lengths corresponding to a visual extinction of $20 \mathrm{mag}$. The symbols are the same as in Fig. 4.

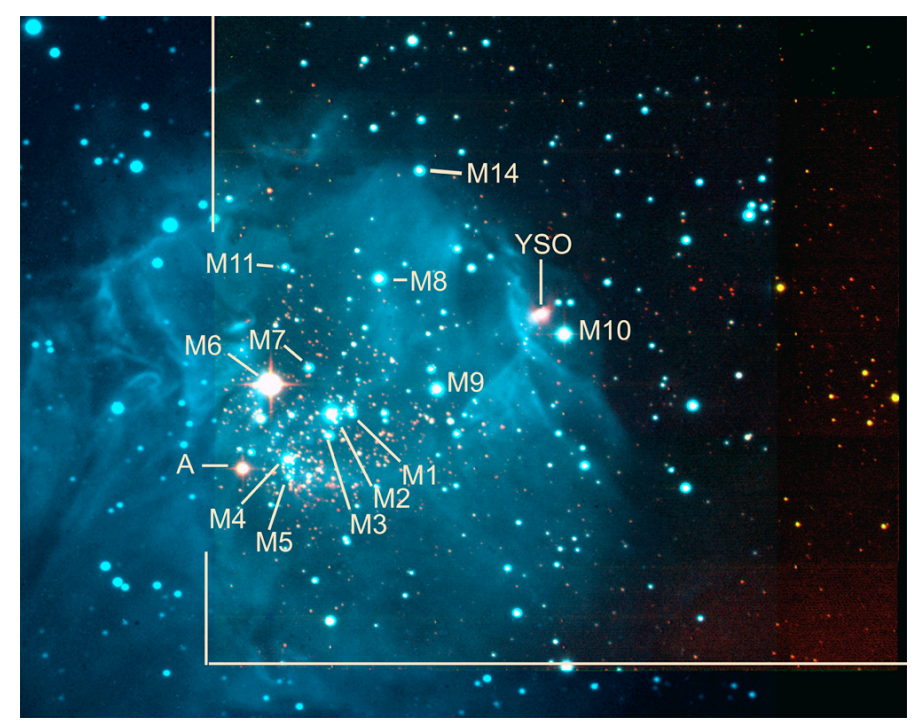

Fig. 6. Identification of a few objects discussed in the text. The underlying image is a colour composite of the [S II] frame (blue) and of the $K$ frame (orange). The lines indicate the limits of our $J H K$ frames.

\section{Molecular observations}

\subsection{Observations}

In March 2006 we observed the emission of the molecular gas associated with $\mathrm{Sh} 2-212$, in the ${ }^{12} \mathrm{CO}$ and ${ }^{13} \mathrm{CO} J=2 \rightarrow 1$ lines using the IRAM 30-m telescope (Pico Veleta, Spain). We mapped an area of $16^{\prime} \times 16^{\prime}$ with the HERA heterodyne array (Schuster et al. 2004). HERA has nine dual polarization pixels. The elements of the array are arranged in a $3 \times 3$ matrix, with a separation of $24^{\prime \prime}$ on the sky between adjacent elements. The 
Table 4. Summary of millimetre line observations.

\begin{tabular}{lcccc}
\hline \hline Line & $\begin{array}{c}\text { Frequency } \\
(\mathrm{GHz})\end{array}$ & $\begin{array}{c}\text { Beam width } \\
(\operatorname{arcsec})\end{array}$ & $B_{\text {eff }}$ & $F_{\text {eff }}$ \\
\hline $\mathrm{CS} J=2 \rightarrow 1$ & 97.98097 & 24 & 0.77 & 0.95 \\
$\mathrm{CS} J=3 \rightarrow 2$ & 146.96905 & 16 & 0.69 & 0.93 \\
$\mathrm{C}^{18} \mathrm{O} J=2 \rightarrow 1$ & 219.56038 & 11 & 0.53 & 0.90 \\
${ }^{13} \mathrm{CO} J=2 \rightarrow 1$ & 220.39872 & 11 & 0.52 & 0.90 \\
${ }^{12} \mathrm{CO} J=2 \rightarrow 1$ & 230.53800 & 11 & 0.52 & 0.90 \\
$\mathrm{CS} J=5 \rightarrow 4$ & 244.93563 & 10 & 0.54 & 0.91 \\
\hline
\end{tabular}

beam size of the telescope is $\sim 11^{\prime \prime}$ at these frequencies (Table 4 ). The data were acquired by drifting the telescope in right ascension ("on-the-fly") in the frequency switching mode. The beam pattern on the sky was rotated by 18.5 degrees with respect to the right ascension axis by means of a K-mirror mounted between the Nasmyth focal plane and the cryostat of the heterodyne array. When drifting the telescope in right ascension, two adjacent rows are separated by $8^{\prime \prime}$, which results in a map slightly under-sampled in declination (the Nyquist sampling step is 5".5). Details about the HERA array and the K-mirror can be found at http: //www . iram. fr/IRAMES/index.htm

We used the WILMA digital autocorrelator, with a spectral resolution of $78 \mathrm{kHz}$, as a spectrometer; the resolution was later degraded to obtain a velocity resolution of $0.2 \mathrm{~km} \mathrm{~s}^{-1}$. The observing conditions were typical for the time of the year, with system temperatures of $400 \mathrm{~K}$ to $500 \mathrm{~K}$. Pointing, which was checked every 90 min by scanning across nearby quasars, was found to be stable to better than $2^{\prime \prime}$.

Supplementary observations in the $\mathrm{C}^{18} \mathrm{O} J=2 \rightarrow 1$ and the CS $J=2 \rightarrow 1 J=3 \rightarrow 2$ and $J=5 \rightarrow 4$ transitions were carried out towards the condensations identified in the HERA maps, using the "standard" heterodyne receivers at the IRAM 30-m telescope. The weather conditions were very good, with system temperatures of $145 \mathrm{~K}$ and $260 \mathrm{~K}$ at $3 \mathrm{~mm}$ and $1.3 \mathrm{~mm}$ respectively.

The ${ }^{12} \mathrm{CO}$ and ${ }^{13} \mathrm{CO}$ emission extends over scales of several arcmin (Fig. 7), comparable to or larger than the first and second error beam of the IRAM telescope (see Table 1 in Greve et al. 1998). Hence the main-beam temperature scale is not a good approximation to the intrinsic ${ }^{12} \mathrm{CO}$ and ${ }^{13} \mathrm{CO}$ line brightnesses. The antenna temperature scale $T_{\mathrm{a}}^{*}$ is a better approximation and we express the $\mathrm{CO}$ and ${ }^{13} \mathrm{CO}$ fluxes in this unit. On the other hand, the $\mathrm{C}^{18} \mathrm{O}$ emission is much more compact, and the mainbeam brightness temperature scale is a reasonable approximation to the intrinsic line brightness. We adopt a value of 0.53 for the main-beam efficiency at the frequency of the $\mathrm{C}^{18} \mathrm{O}$ line.

Table 4 gives a summary of the millimetre observations and the efficiencies used.

\subsection{Distribution and kinematics}

In all the maps presented hereafter, the coordinates are expressed in arcsecond offsets with respect to the MSX point source. The distribution of the integrated ${ }^{12} \mathrm{CO}$ emission as a function of velocity is shown in Fig. 7. The CO line traces a diffuse filamentary cloud which extends southeast-northwest. The velocity of this filament is between -36 and $-37 \mathrm{~km} \mathrm{~s}^{-1}$, hence a few kilometres per second more positive than that of the ionized gas. A bright and thin half-ring structure of molecular gas is very clearly associated with the photo-dissociation region. This half-ring follows the ring of MSX emission at $8.3 \mu \mathrm{m}$ which surrounds the brightest part of the ionized region. It lies at the back of the H II region, as no corresponding extinction of the optical nebular emission is observed in its direction. This is consistent with the observed velocity field, which shows that the molecular gas ring is redshifted with respect to the molecular filament and the ionized gas.

The ring is fragmented into at least five condensations (Fig. 8). From west to east we have condensation 1, observed in the direction of the MSX point source and the UC H II region (see Sect. 5), with a mean velocity of $-35.5 \mathrm{~km} \mathrm{~s}^{-1}$; condensations 2 and 3 with a velocity of $-33.5 \mathrm{~km} \mathrm{~s}^{-1}$; condensation 4 with a velocity of $-32.5 \mathrm{~km} \mathrm{~s}^{-1}$. Further to the east lies condensation 5 with a velocity of $-37.5 \mathrm{~km} \mathrm{~s}^{-1}$.

The velocity varies along the half-ring structure: condensations 1 and 5, situated on opposite ends of this structure, have velocities not very different from that of the filament; the halfring, at the rear of the Sh2-212 H II region, is expanding with a velocity of a few kilometres per second with respect to the filament. The ionized gas flows away from the filament in the opposite direction (see Fig. 13).

Figure 8 compares the ${ }^{13} \mathrm{CO}$ emission, especially the locations of the condensations, with the [S II] emission of the ionized gas. Figure 8 shows that the bright rim present at the northwest border of Sh2-212, which harbours the UC H II region and star No. 228, is the ionized border of condensation 1 . Thus condensation 1 appears as the remains of the parental core in which the massive YSO No. 228 formed and subsequently ionized a UC H II region. Furthermore, several molecular substructures have counterparts in the [S II] image, as substructures of the ionization front. For example, condensation 5 is situated behind an IF traced by [S II] emission.

\subsection{Physical conditions}

\subsubsection{The kinetic temperature}

${ }^{12} \mathrm{CO}$ brightness temperatures of $20-30 \mathrm{~K}$ are observed along the molecular gas ring and the cores. Towards $\mathrm{C} 1$, the maximum of ${ }^{12} \mathrm{CO}$ brightness is observed at the offset position $\left(-3^{\prime \prime},-1^{\prime \prime}\right)$, where $T_{\mathrm{a}}^{*}=24 \mathrm{~K}$. This implies a kinetic temperature $T_{\mathrm{k}} \approx 27 \mathrm{~K}$. The peak brightness temperatures in the ${ }^{13} \mathrm{CO}$ and $\mathrm{C}^{18} \mathrm{O} J=2 \rightarrow 1$ transitions are $11 \mathrm{~K}$ and $1.5 \mathrm{~K}$, compatible with opacities $\tau_{13}=0.58$ and $\tau_{18}=0.08$, respectively, adopting standard relative abundances $\left[{ }^{12} \mathrm{CO}\right] /\left[{ }^{13} \mathrm{CO}\right]=60$ and $\left[{ }^{13} \mathrm{CO}\right] /\left[\mathrm{C}^{18} \mathrm{O}\right]=8$. Hence the ${ }^{13} \mathrm{CO} J=2 \rightarrow 1$ transition is moderately optically thin. The $\mathrm{C}^{18} \mathrm{O}$ line traces a gas column density $N\left(\mathrm{H}_{2}\right)=4.3 \times 10^{21} \mathrm{~cm}^{-2}$ at the intensity peak of $\mathrm{C} 1$. Calculations of the $\mathrm{C}^{18} \mathrm{O}$ line excitation in the large velocity gradient (LVG) approximation show that the opacity of the line is consistent with a gas kinetic temperature of $30 \mathrm{~K}$, as estimated above. A similar conclusion was derived for C3. Hence we adopt a kinetic temperature of $30 \mathrm{~K}$ for the condensations, in what follows.

Inside the filamentary cloud, away from the HII region, the ${ }^{12} \mathrm{CO}$ and ${ }^{13} \mathrm{CO} J=2 \rightarrow 1$ brightness temperatures are much lower, typically a few kelvins for both lines. At the offset position $\left(-120^{\prime \prime},+80^{\prime \prime}\right)$, we measure $T_{B}\left({ }^{12} \mathrm{CO}\right)=8.2 \mathrm{~K}$ and $T_{B}\left({ }^{13} \mathrm{CO}\right)=2.1 \mathrm{~K}$, which implies an opacity $\tau_{13}=0.32$, adopting the same standard abundance ratio. Both line intensities and opacities are accounted for by a gas layer of column density $N\left(\mathrm{H}_{2}\right)=9 \times 10^{20} \mathrm{~cm}^{-2}$ at about $14 \mathrm{~K}$.

\subsubsection{Masses and densities}

The masses of the molecular gas fragments were derived from the ${ }^{13} \mathrm{CO}$ data, in the optically thin limit. For condensation $\mathrm{C} 1$ 


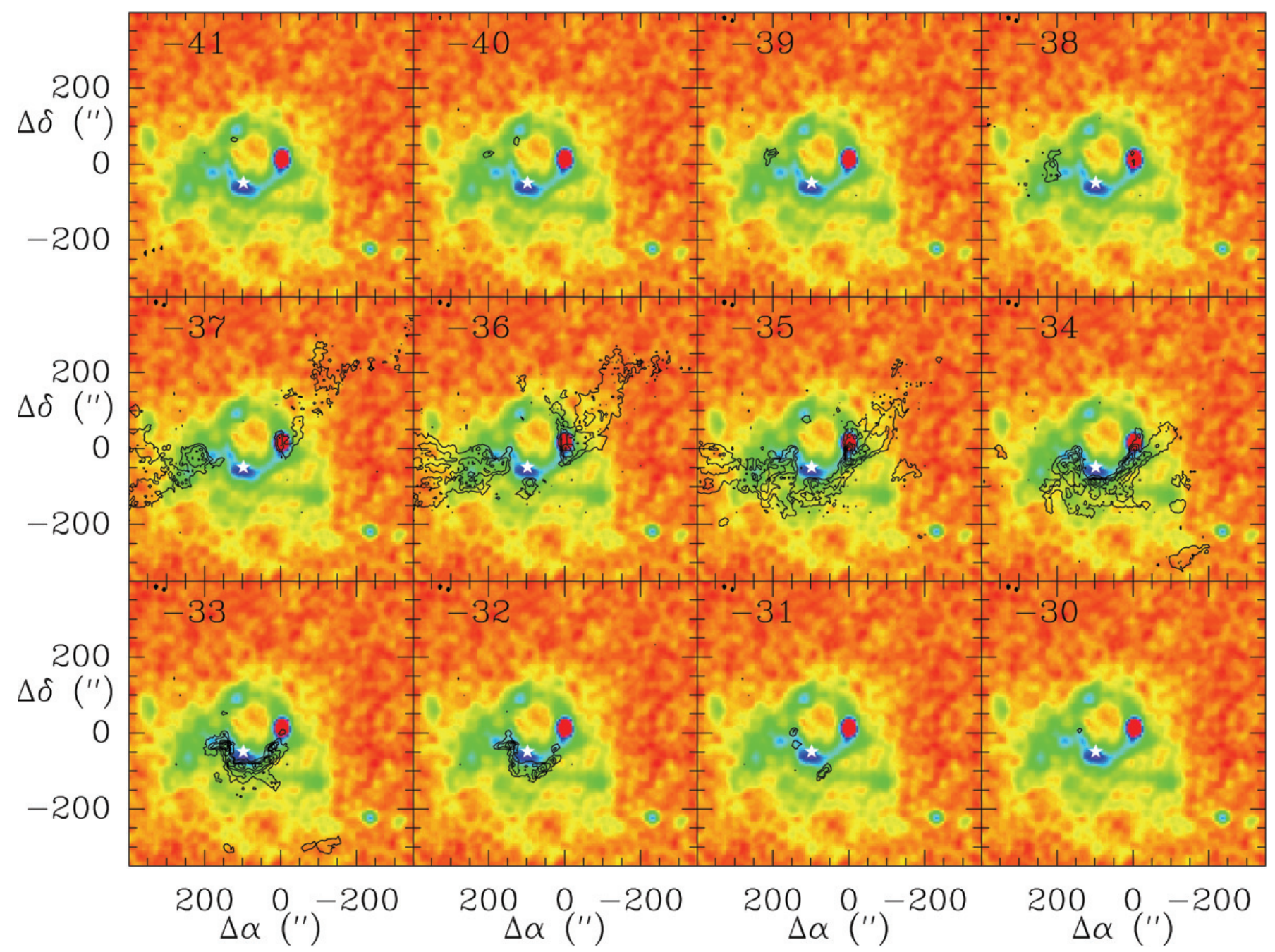

Fig. 7. Channel maps of the ${ }^{12} \mathrm{CO} J=2 \rightarrow 1$ emission as observed with HERA and integrated over velocity intervals of $1 \mathrm{~km} \mathrm{~s}^{-1}$; the central velocity of each bin is marked in the upper left corner of each panel. The first contour is at $1 \mathrm{~K} \mathrm{~km} \mathrm{~s}^{-1}$ and the contour interval is $2 \mathrm{~K} \mathrm{~km} \mathrm{~s}{ }^{-1}$. The coloured background is the MSX emission at $8.3 \mu \mathrm{m}$. The 0,0 position is that of the MSX point source (Table 1).

the contour at half power of the ${ }^{13} \mathrm{CO} J=2 \rightarrow 1$ emission delineates a condensation of $56^{\prime \prime} \times 28^{\prime \prime}$, oriented north-south, centred at offset position $\left(-6^{\prime \prime},-1^{\prime \prime}\right)$. The total mass of the condensation is obtained by integrating over the $20 \%$ peak contour, from which we determine $M=220 M_{\odot}$. A similar procedure was applied to the five condensations. The results are summarised in Table 5.

The half shell of collected molecular material has a mass $\leq 720 M_{\odot}$, as estimated by integrating the whole ${ }^{13} \mathrm{CO} J=2 \rightarrow 1$ emission in the ring.

The mean density in a fragment, $n\left(\mathrm{H}_{2}\right)$, is derived as $\left.N\left(\mathrm{H}_{2}\right) / \sqrt{(} a b\right)$ where $a$ and $b$ are the major and minor axes of the condensation. We have taken into account the dilution in the main beam, by applying a correction factor $(a b) /\left(a b+\theta_{\text {beam }}^{2}\right)$, where $\theta_{\text {beam }}$ is the beam width. These mean densities are given in Table 5.

Emission of the high-density gas was traced by the millimetre lines of CS (Fig. 9). The emission of the lower transition $J=2 \rightarrow 1$ is detected along the filament, whereas the $J=3 \rightarrow 2$ and $J=5 \rightarrow 4$ emissions are more compact. The $J=5 \rightarrow 4$ transition was detected only in $\mathrm{C} 1$ and $\mathrm{C} 4$, arising from a small unresolved region. The $J=2 \rightarrow 1$ and $J=3 \rightarrow 2$ lines intensities are typically 1 to $2 \mathrm{~K}$ at the brightness peak of the condensations. The lines are typically $1 \mathrm{~km} \mathrm{~s}^{-1}$ wide.
Estimates of the $\mathrm{H}_{2}$ density in the fragments were obtained by modelling the millimetre CS line emission, in the LVG approximation. Analysis of the $J=2 \rightarrow 1$ and the $J=3 \rightarrow 2$ transitions at the brightness peak indicate typical densities $n\left(\mathrm{H}_{2}\right) \simeq$ $2-4 \times 10^{5} \mathrm{~cm}^{-3}$ in the cores, and up to $1.0 \times 10^{6} \mathrm{~cm}^{-3}$ towards $\mathrm{C} 4$. Note that these densities are much higher than the mean densities estimated from the $\mathrm{C}^{18} \mathrm{O}$ emission; thus the $\mathrm{CS}$ material has a small filling factor.

\section{Radio observations}

We observed Sh2-212, in the direction of the MSX point source, with the Very Large Array (VLA) on 2005 November 16. We searched for emission from methanol at $44 \mathrm{GHz}(7 \mathrm{~mm})$, water at $22 \mathrm{GHz}(1.3 \mathrm{~cm})$, ammonia in the $(1,1)$ and $(2,2)$ lines at $23 \mathrm{GHz}$, and neutral hydrogen at $21 \mathrm{~cm}$. The array was in its most compact configuration, providing spatial resolutions of $1^{\prime \prime} .5,3 . .3$, and $45^{\prime \prime}$ in the $7 \mathrm{~mm}, 1.3 \mathrm{~cm}$, and $21 \mathrm{~cm}$ bands, respectively. Each molecular transition was observed with a distinct combination of bandwidth and number of channels, resulting in spectral resolutions of $0.3 \mathrm{~km} \mathrm{~s}^{-1}$ for methanol and water, and $0.6 \mathrm{~km} \mathrm{~s}^{-1}$ for ammonia. For all three molecules the total bandwidth corresponds to about $40 \mathrm{~km} \mathrm{~s}^{-1}$, and is centred at $-35 \mathrm{~km} \mathrm{~s}^{-1}$. Further observational details will be provided in a 

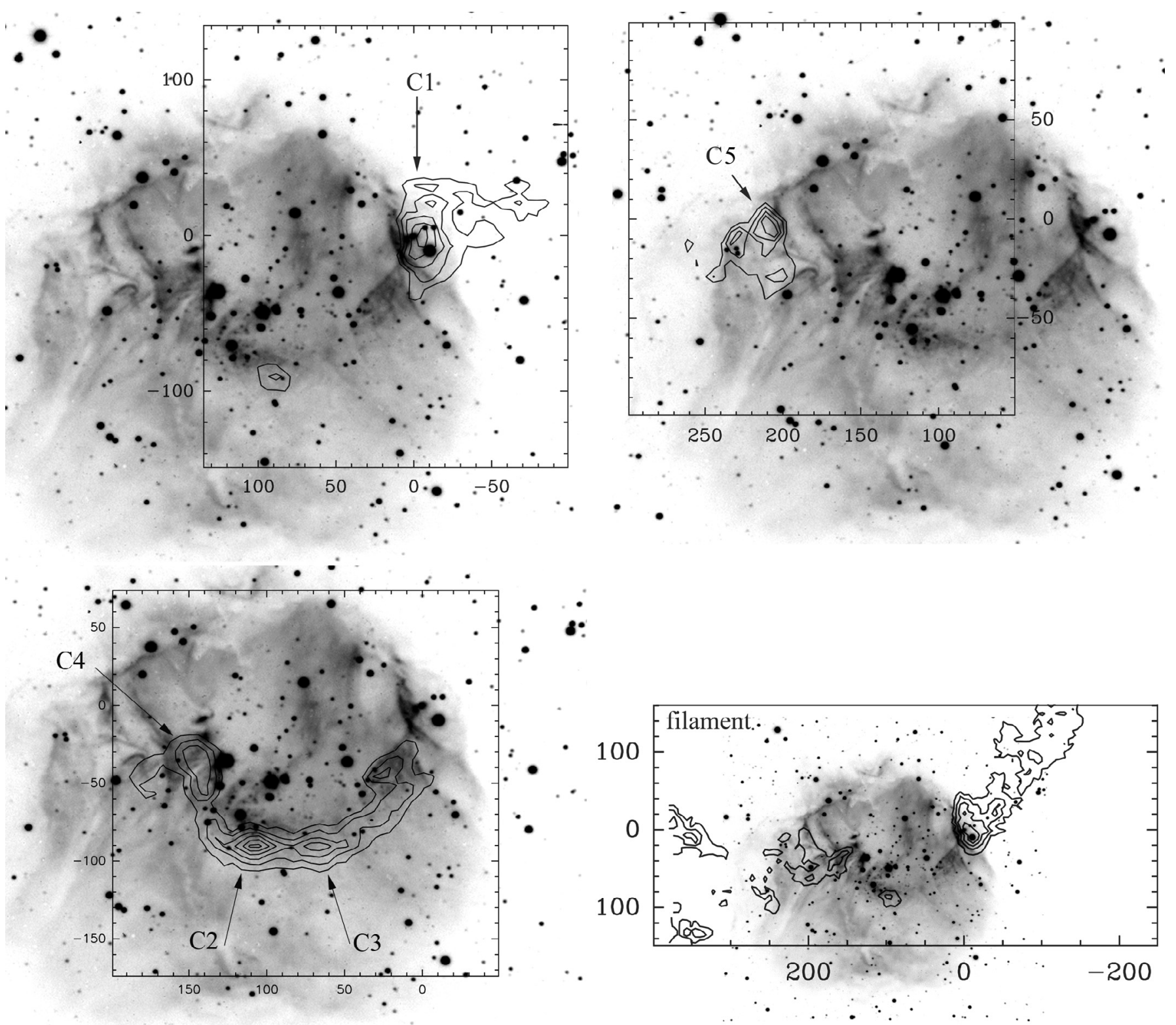

Fig. 8. Top left: condensation $\mathrm{C} 1$, showing the ${ }^{13} \mathrm{CO}(2-1)$ emission integrated between $-36.1 \mathrm{~km} \mathrm{~s}^{-1}$ and $-35.1 \mathrm{~km} \mathrm{~s}^{-1}$. The first contour and the step correspond respectively to $20 \%$ and $15 \%$ of the peak brightness $\left(9.6 \mathrm{~K} \mathrm{~km} \mathrm{~s}^{-1}\right)$. Bottom Left: condensations C2, C3, and C4, showing the ${ }^{13} \mathrm{CO}(2-1)$ emission integrated between $-34.0 \mathrm{~km} \mathrm{~s}^{-1}$ and $-32.7 \mathrm{~km} \mathrm{~s}^{-1}$. The first contour and the step are respectively $20 \%$ and $15 \%$ of the peak brightness $\left(15.7 \mathrm{~K} \mathrm{~km} \mathrm{~s}^{-1}\right)$. Top right: condensation $\mathrm{C} 5$, showing the ${ }^{13} \mathrm{CO}(2-1)$ emission integrated between $-38.4 \mathrm{~km} \mathrm{~s}^{-1}$ and $-36.6 \mathrm{~km} \mathrm{~s}^{-1}$. The first contour and the step are respectively $35 \%$ and $15 \%$ of the peak brightness $\left(3.8 \mathrm{~K} \mathrm{~km} \mathrm{~s}^{-1}\right)$. Bottom right: filament, with the ${ }^{13} \mathrm{CO}(2-1)$ emission integrated between $-36.8 \mathrm{~km} \mathrm{~s}^{-1}$ and $-35.9 \mathrm{~km} \mathrm{~s}^{-1}$. The peak is at $6.6 \mathrm{~K} \mathrm{~km} \mathrm{~s}^{-1}$. The first contour and the step are respectively $20 \%$ and $20 \%$ of the peak brightness.

forthcoming paper. We did not detect any of the molecular transitions to the $5 \sigma$ levels of 90,33 , and $10 \mathrm{mJy} \mathrm{beam}^{-1}$ for the methanol, water, and ammonia lines, respectively. The neutral hydrogen line at $21 \mathrm{~cm}$ was detected, and is discussed below.

The absence of $44 \mathrm{GHz}$ methanol masers is not necessarily surprising. Methanol masers are known to be associated with high-mass star formation (e.g., Ellingsen 2006); however, type II methanol masers (such as those producing the 6.7 and $12.2 \mathrm{GHz}$ lines) are thought to be pumped by the radiation field of YSOs, and hence are closely linked with the star formation process. Type I masers (such as those producing the $44 \mathrm{GHz}$ line) are thought to be collisionally pumped, and may not be directly associated with massive YSOs. More surprising is the absence of water masers, which are nearly ubiquitous in star-forming regions. Water masers are known to be variable, and a possible explanation for our non-detection is that such masers are present but currently quiescent. Other explanations for the non-detection may have implications for the star formation process. For example, water masers are typically thought to arise in outflow and/or accretion processes, which may be absent in YSO No. 228.

Our $5 \sigma$ detection level of $10 \mathrm{mJy}^{\mathrm{beam}}{ }^{-1}$ for the $(1,1)$ and $(2,2)$ ammonia lines allows us to estimate an upper limit for the gas column density. Assuming optically thin emission, with a $30 \mathrm{~K}$ excitation temperature, and a beam filling factor of one, our observations could detect an ammonia column density of about $10^{14} \mathrm{~cm}^{-2}$. Adopting an ammonia abundance of $10^{-8}$ indicates a detection limit of $\mathrm{H}_{2}$ column density of about $10^{22} \mathrm{~cm}^{-2}$. This is marginally higher than the several times $10^{21} \mathrm{~cm}^{-2}$ column 
Table 5. Properties of the molecular gas condensations.

\begin{tabular}{ccccccccc}
\hline \hline Condensation & $\begin{array}{c}\text { Offset position } \\
(")\end{array}$ & $\begin{array}{c}\Delta v^{1} \\
\left(\mathrm{~km} \mathrm{~s}^{-1}\right)\end{array}$ & $\begin{array}{c}\text { Core dimensions } \\
\left({ }^{\prime \prime}\right)\end{array}$ & $\begin{array}{c}N\left(\mathrm{H}_{2}\right)^{\text {peak }, 1} \\
\left(\mathrm{~cm}^{-2}\right)\end{array}$ & $\begin{array}{c}n\left(\mathrm{H}_{2}\right)^{1} \\
\left(\mathrm{~cm}^{-3}\right)\end{array}$ & $\begin{array}{c}N(\mathrm{CS}) \\
\left(\mathrm{cm}^{-2}\right)\end{array}$ & $\begin{array}{c}n\left(\mathrm{H}_{2}\right)^{\text {peak }, 2} \\
\left(\mathrm{~cm}^{-3}\right)\end{array}$ & $\begin{array}{c}\text { Fragment mass } \\
\left(M_{\odot}\right)\end{array}$ \\
\hline 1 & $(-6,-5)$ & 1.1 & $25 \times 8$ & $4.3(21)$ & $1.0(4)$ & $6.5(12)$ & $4(5)$ & 220 \\
2 & $(+108,-90)$ & 1.2 & $25 \times 12$ & $6.7(21)$ & $1.0(4)$ & $3.8(12)$ & $4(5)$ & 63 \\
3 & $(+68,-90)$ & 1.2 & $25 \times 10$ & $4.3(21)$ & $8.2(3)$ & $2.7(12)$ & $1.8(5)$ & 45 \\
4 & $(+136,-30)$ & 1.0 & $15 \times 10$ & $7.2(21)$ & $2.5(4)$ & $1.2(13)$ & $1.0(6)$ & 61 \\
5 & $(+210,-5)$ & $2.0^{3}$ & $16 \times 16$ & $2.7(21)$ & - & - & - & 38 \\
\hline
\end{tabular}

${ }^{1}$ Determined from $\mathrm{C}^{18} \mathrm{O}$ data. ${ }^{2}$ Determined from $\mathrm{CS}$ data. ${ }^{3}$ From ${ }^{13} \mathrm{CO}$ data.

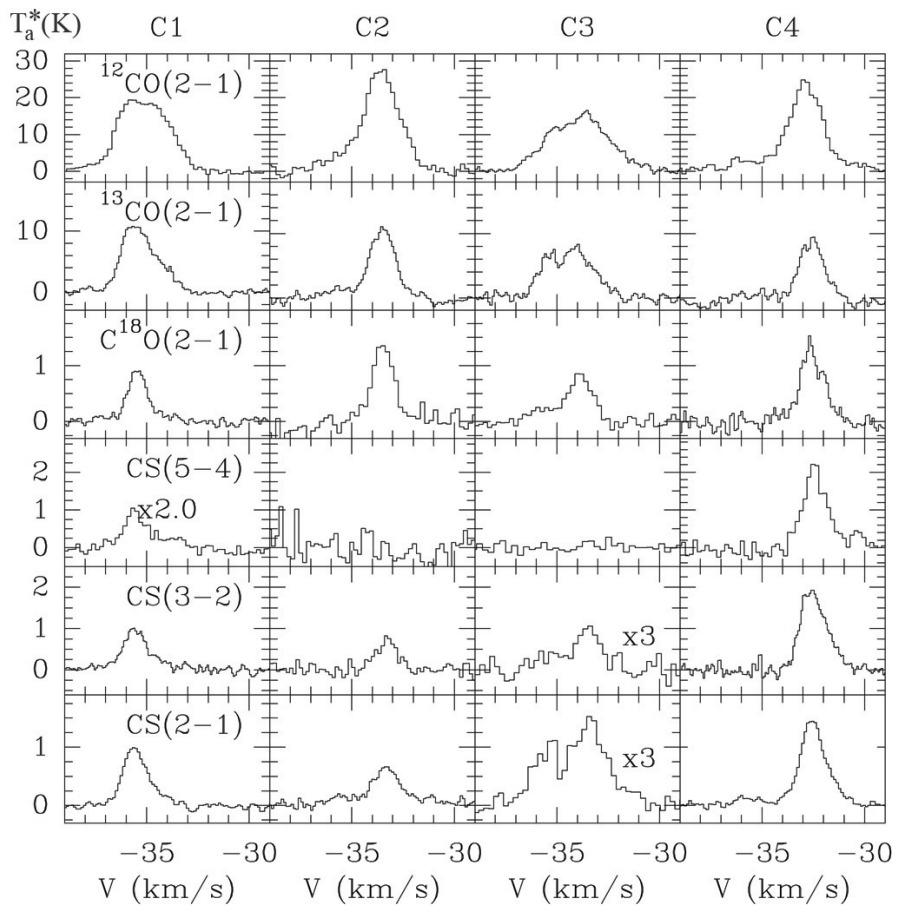

Fig. 9. Molecular lines observed towards the molecular fragments $\mathrm{C} 1$ to C4.

densities reported in Table 5; thus our non-detection of ammonia is consistent with the column densities inferred from the $\mathrm{CO}$ observations. Hot molecular cores, a common tracer of young, high-mass star formation, with column densities of $10^{23}$ $10^{24} \mathrm{~cm}^{-2}$, are clearly absent.

Centimetre continuum data of Sh2-212 were obtained from the VLA data archive of programmes AF346 and AR390, at $1.46 \mathrm{GHz}$ and $8.69 \mathrm{GHz}$ respectively. These data were calibrated and imaged using standard procedures for continuum data. The AF346 observations were made in 1998 in the C and B configurations. The lower resolution $\left(16^{\prime \prime} \times 13^{\prime \prime}\right) \mathrm{C}$-array data imaged both the Sh2-212 region and a compact source to the northwest. The resolution was too low for a reliable determination of the compact source's parameters; the higher resolution $\left(5^{\prime \prime} \times 4^{\prime \prime}\right)$ B-array data were used for this purpose. The AR390 observations were made in 1997 in the D configuration, and provided an angular resolution of $10^{\prime \prime} \times 7^{\prime \prime}$. Sh2-212 was too large to be imaged by these data, but the compact source parameters were reliably determined to be $11 \pm 1 \mathrm{mJy}$ at $1.46 \mathrm{GHz}$ and $7.6 \pm 1 \mathrm{mJy}$ at $8.69 \mathrm{GHz}$. Gaussian fits to the source size and position yield deconvolved major and minor axes of $3^{\prime \prime}$, and a J2000 position $04^{\mathrm{h}} 40^{\mathrm{m}} 27^{\mathrm{s}} \cdot 2,+50^{\circ} 28^{\prime} 29^{\prime \prime}$, i.e. coincident with the MSX source G155.3319+02.5989 (see Table 1). Although no ammonia was detected in our 2005 observations, we used the central $75 \%$ of

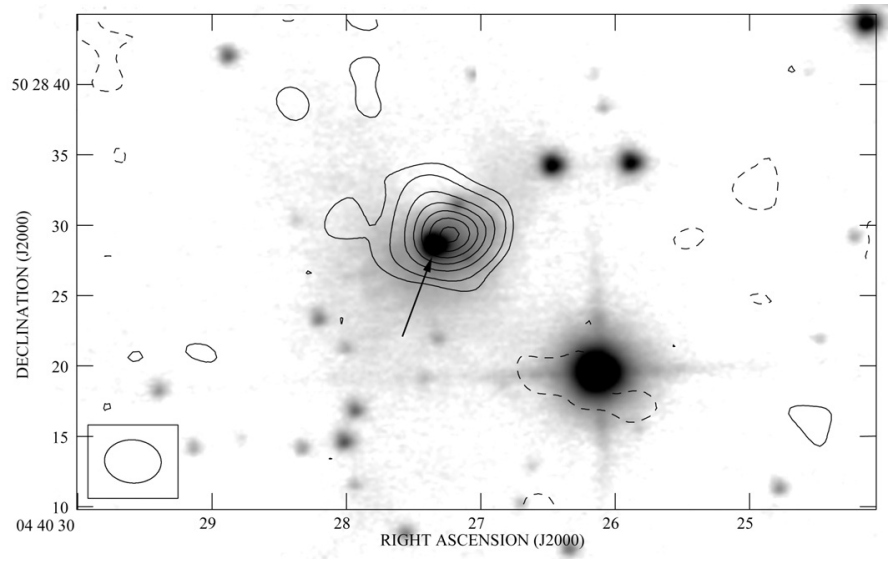

Fig. 10. Radio continuum emission (contours) superimposed on the $H$ image (grey scale). Star No. 228 is indicated by an arrow. The contours show $1.3 \mathrm{~cm}$ continuum emission from the UC H II region coinciding in direction with the MSX point source and the star. The angular resolution of the radio image is 3 .' 3 (indicated in the lower left corner) and the contour levels are $-15,15,20,25,30,40,45$ mJy beam $^{-1}$.

the $3.1 \mathrm{MHz}$ bandwidth to form a $1.3 \mathrm{~cm}$ continuum image, presented in Fig. 10. The compact source was detected in this image, with a flux density of $8.9 \pm 1.2 \mathrm{mJy}$.

For the UC H II region, the flux densities at 1.46, 8.69, and $23.7 \mathrm{GHz}$ show a flat spectrum, indicative of an optically thin $\mathrm{H}$ II region. All three observations indicate an ionizing photon flux of $\log N_{\mathrm{Lyc}}>46.5$, or the equivalent of a B1 or earlier star (Smith et al. 2002). The $3^{\prime \prime}$ size corresponds to $0.095 \mathrm{pc}$ at a distance of $6.5 \mathrm{kpc}$. Assuming a spherical H II region of this diameter, the flux densities indicate an rms electron density of $3.2 \times 10^{3} \mathrm{~cm}^{-3}$, with a total mass of ionized gas of $0.05 M_{\odot}$.

Sh2-212 was also observed with the VLA in the $21 \mathrm{~cm}$ line of neutral hydrogen. The observations were made with a $1.56 \mathrm{MHz}\left(330 \mathrm{~km} \mathrm{~s}^{-1}\right)$ bandwidth and 255 channels of $6.1 \mathrm{kHz}$ $\left(1.3 \mathrm{~km} \mathrm{~s}^{-1}\right)$ each. Data reduction followed standard VLA spectral line procedures. After the external flux and phase calibration, continuum emission was subtracted from the $u v$ data and an image cube was formed. Very extended Hi emission was present in the field while the H I emission on size scales similar to those of Sh2-212 was relatively weak. To optimise the imaging toward the H I associated with Sh2-212, the data were re-imaged, removing the shortest $0.3 \mathrm{k} \lambda$ baselines (to suppress the extended emission) and averaging adjacent channels (to improve the signal-to-noise ratio). The resulting image cube has an angular resolution of $50^{\prime \prime} \times 41^{\prime \prime}$ and a spectral resolution of $2.6 \mathrm{~km} \mathrm{~s}^{-1}$. H I emission was found in three adjacent channels, from $-40 \mathrm{~km} \mathrm{~s}^{-1}$ to $-48 \mathrm{~km} \mathrm{~s}^{-1}$. Three distinct H I condensations were found, all lying (in projection) at the edge of the HII region. A contour plot of this emission is shown in Fig. 11. Using the peak brightness temperature (in a $45^{\prime \prime}$ beam) 


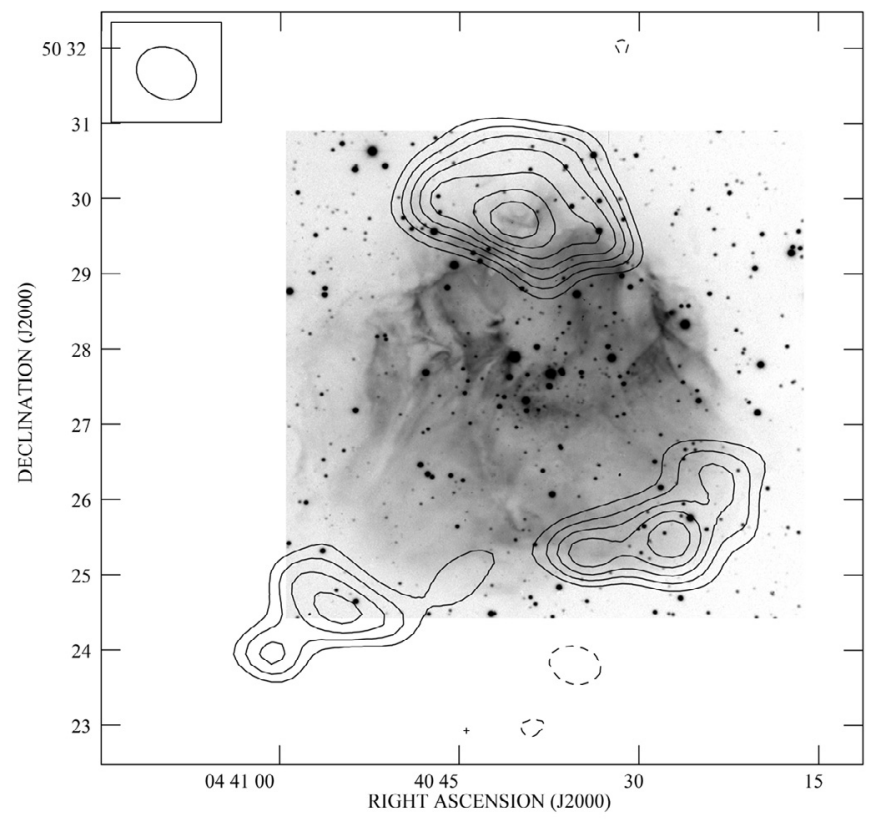

Fig. 11. H I emission associated with Sh2-212, integrated between $-40 \mathrm{~km} \mathrm{~s}^{-1}$ and $-48 \mathrm{~km} \mathrm{~s}^{-1}$. The radio angular resolution $\left(50^{\prime \prime} \times 41^{\prime \prime}\right)$ is indicated in the upper left corner. Contour levels are $-15,15,20,25$, ... $50 \mathrm{mJy}$ beam $^{-1} \mathrm{~km} \mathrm{~s}^{-1}$. The H I contours are superimposed on the [S II] image.

we can calculate the column density over the central $1.4 \mathrm{pc}$ of each clump. Assuming optically thin emission, so that the observed line temperature is approximately equal to the spin temperature times the optical depth, we calculate the column density as $N_{\mathrm{HI}}\left(\mathrm{cm}^{-2}\right)=1.82 \times 10^{18}\left(T_{L} / \mathrm{K}\right)\left(\Delta V / \mathrm{km} \mathrm{s}^{-1}\right)$. All three clumps have peak line temperatures of $20-25 \mathrm{~K}$, and linewidths of $4-5 \mathrm{~km} \mathrm{~s}^{-1}$. Hence, for all three we find column densities of about $1.5 \times 10^{20} \mathrm{~cm}^{-2}$ (within a factor of two). Assuming a spherical geometry for the central region of each clump implies hydrogen densities of about $35 \mathrm{~cm}^{-3}$.

\section{Discussion}

\subsection{A massive young stellar object exciting a UC H II region}

Star No. 228, associated with a reflection nebulosity, is a massive young stellar object:

- It is a luminous near-IR source with a near-IR excess indicative of the presence of nearby dust, probably associated with a disk (Lada \& Adams 1992).

- Its spectral energy distribution (SED) rises strongly in the IR. Table 6 gives the flux measurements between $1.25 \mu \mathrm{m}$ and $21.3 \mu \mathrm{m}$. We have used the Web-based SED fitting tool of Robitaille et al. (2007; http://caravan.astro.wisc. edu/protostars/) to interprete that of YSO No. 228 and its associated nebulosity. Several parameters of the model are not well constrained, but some are. A strong conclusion is that the central stellar object is hot $\left(T_{*}=30000 \pm 1000 \mathrm{~K}\right)$ and massive $\left(M_{*} \sim 14 M_{\odot}\right)$, and that the YSO is luminous (total luminosity 14000-20000 $L_{\odot}$ ); the disk and the envelope have similar luminosities (more uncertain). Also, the YSO is seen edge-on (inclination angle $\sim 87^{\circ}$ ). Figure 12 shows the SED of YSO No. 228 and the five best-fitting models.

Figure 10 shows the radio-continuum emission of the UC H II region at $1.3 \mathrm{~cm}$, as contours superimposed on an $H$ image. Star
Table 6. Spectral energy distribution of star No. 228, a massive YSO.

\begin{tabular}{ccl}
\hline \hline $\begin{array}{c}\text { Wavelength } \\
(\mu \mathrm{m})\end{array}$ & $\begin{array}{c}F_{v} \\
(\mathrm{Jy})\end{array}$ & Origin \\
\hline 1.215 & $0.629 \times 10^{-2}$ & $J$ star alone \\
1.215 & $1.268 \times 10^{-2}$ & $J$ star + nebulosity \\
1.65 & $1.049 \times 10^{-2}$ & $H$ star alone \\
1.65 & $2.006 \times 10^{-2}$ & $H$ star + nebulosity \\
2.18 & $1.268 \times 10^{-2}$ & $K$ star alone \\
2.18 & $3.112 \times 10^{-2}$ & $K$ star + nebulosity \\
8.28 & 2.27 & MSX star + nebulosity \\
12.13 & 2.91 & MSX star + nebulosity \\
14.65 & 4.94 & MSX star + nebulosity \\
21.3 & 35.34 & MSX star + nebulosity \\
\hline
\end{tabular}

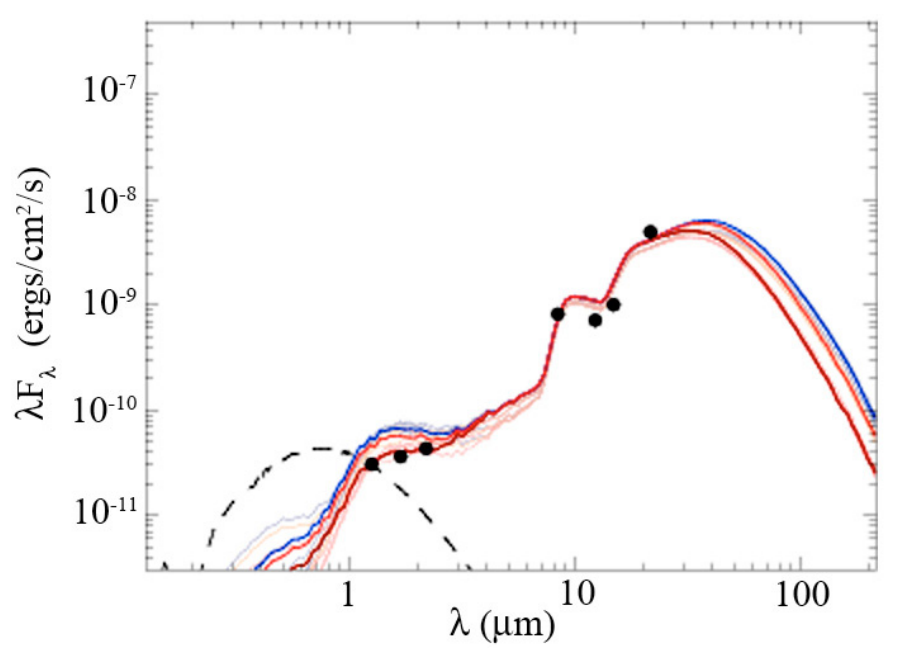

Fig. 12. Spectral energy distribution of star No. 228, a massive YSO. Filled circles are the fluxes listed in Table 6 . The five best fitting models obtained using the web-based tool of Robitaille et al. (2007) are presented.

No. 228 lies at the centre of the UC H II region (see also Table 1). Thus star No. 228 is very probably the exciting star of the UC H II region.

The SED of object No. 228 indicates that it is probably in an evolutionary stage between Class I and Class II, with both an envelope and a disk. This object is consistent with the evolutionary models of massive stars, formed by accretion, as described by Beech \& Mitalas (1994) and by Bernasconi \& Maeder (1996). This object of $\sim 14 M_{\odot}$ has reached the main sequence, and hence is burning hydrogen in its centre. But it is still accreting material and increasing its mass. Presently its accretion rate is not high enough to prevent the formation of an ionized region (Walmsley 1995).

This massive YSO, which is observed at its place of birth inside the parental condensation, does not seem to belong to a populous cluster. Only three low-brightness stars are observed nearby, at less than $0.11 \mathrm{pc}$ (with $K$ magnitudes $\geq 16.1$ ). It is therefore a good candidate for being a massive star born either in isolation or in a very small cluster. As such, it deserves further high-resolution, high-sensitivity imaging and spectroscopic observations to ascertain its nature and to detect any nearby deeplyembedded objects. 


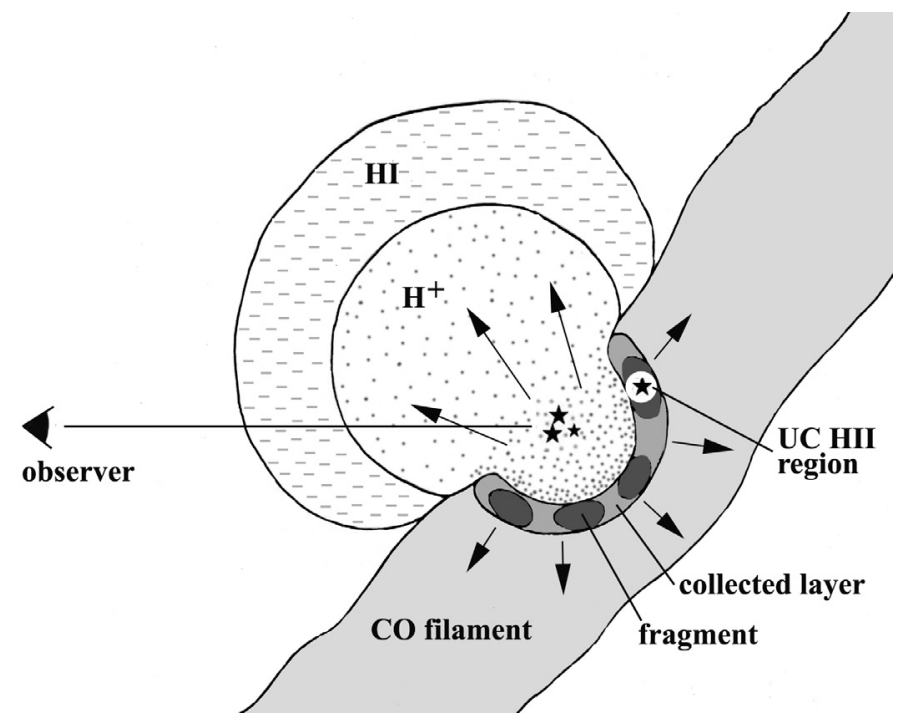

Fig. 13. Morphology of the Sh2-212 complex.

\subsection{The age of the $U C H$ II region}

The UC H II region formed and evolved in condensation 1. Let us assume that it formed in a uniform medium of density $10^{5} \mathrm{~cm}^{-3}$. The exciting star, emitting $10^{46.5}$ ionizing photons per second, very quickly formed an ionized region of radius $0.0046 \mathrm{pc}$, which later expanded. According to Dyson \& Williams (1997, Sect. 7.1.8) the present radius of the UC H II region, $0.0475 \mathrm{pc}$, corresponds to an age of $13500 \mathrm{yr}$, and a density in the ionized gas of $3050 \mathrm{~cm}^{-3}$, which is very close to that which is observed. Also, the pressure equilibrium with the surrounding medium has not yet been reached (the expansion velocity being $\sim 2 \mathrm{~km} \mathrm{~s}^{-1}$ ).

Thus this UC H II region is very young - much younger than Sh2-212 (see Sect. 6.4). Our non-detection of water maser emission is a little surprising, and higher-sensitivity observations are needed.

Note, however, that the age of the $\mathrm{H}$ II region is not that of the massive YSO No. 228, which is much older, having evolved during a long time before being able to ionize the surrounding gas.

\subsection{The morphology of the Sh2-212 complex}

The present distribution of the molecular material indicates that Sh2-212 probably formed in a filament. Was this medium turbulent? One morphological aspect of this region, the perfectly spherical shape of the ionized region, at both optical and radio wavelengths, seems to indicate that the level of turbulence, if any, is low. We suggest (see Fig. 13) that the exciting star of Sh2212 formed inside the molecular filament and that the H II region first expanded inside this filament. When the ionization front reached the border of the filament, the $\mathrm{H}$ II region opened on the outside (a low-density inter-filament medium), thus creating a champagne flow. This explains both the velocity field (the ionized gas flowing away from the molecular cloud, more or less in the direction of the observer, at a few kilometres per second), and the shape of the $\mathrm{H}$ II region and its photodissociation region containing the PAHs (a bright ionized region surrounded by the PAH emission ring observed by MSX, and a more diffuse extension of the ionized gas).

During its supersonic expansion inside the molecular filament, the ionization front was preceded by a shock front on the neutral side, and neutral material accumulated between the two fronts. This collected material forms the thin molecular half-ring which surrounds the brightest part of the H II region. This collected layer forms only half a shell, adjacent to the molecular filament, and is in expansion with a velocity of $4-5 \mathrm{~km} \mathrm{~s}^{-1}$ with respect to the molecular filament. On the other side the H II region is surrounded by low-density atomic material; this is the origin of the HI emission observed at the periphery of Sh2-212 (Fig. 11). This atomic material is receding from the molecular filament with a velocity similar to that of the ionized gas. The low density of this material is confirmed by the very low, if any, local extinction in front of the ionized gas. Such atomic hydrogen rings have been observed in other complexes, for example around the spherical H II region Sh2-219 (Roger \& Leahy 1993; Deharveng et al. 2006, Fig. 4), and around Sh2-217 (Roger \& Leahy 1993; Brand et al., in preparation).

Dale et al. (2005) have simulated the photoionizing feedback of a massive star on a turbulent molecular cloud. Figure 14 shows the striking similarity between the morphologies of the observed Sh2-212 molecular cloud and of the simulated cloud. The right panel - simulation - shows the column density of the neutral gas; the ionized region lies in the central hole. The left panel - observations - shows the ${ }^{12} \mathrm{CO}$ emission integrated over velocity (the intensity is proportional to the column density, except for regions optically thick along the line of sight); here again the ionized region lies inside the central hole. The simulation concerns a turbulent cloud of relatively low density; a more chaotic morphology is obtained in the case of a denser turbulent cloud (Dale et al.).

\subsection{Star formation history}

It is almost impossible to obtain direct evidence of sequential star formation. The age of the evolved Sh2-212 is very uncertain due to our lack of knowledge about the density structure of the original medium in which this $\mathrm{H}$ II region formed and evolved. Only indirect evidence is available.

It is difficult to explain the origin of the thin circular halfring of molecular material which surrounds the brightest part of Sh2-212 other than by material collected during the expansion of this $\mathrm{H}$ II region. It is presently fragmented; at least five condensations are present along the ring. This is a good illustration of the collect and collapse process. The most massive condensation contains a massive young stellar object exciting a UC H II region. This UC H II region is very young - much younger than Sh2-212. Thus massive star-formation has been triggered by the expansion of the Sh2-212 H II region, via the collect and collapse process. Here again this process seems able to form massive objects, as observed on the borders of Sh2-104 (Deharveng et al. 2003) and RCW 79 (Zavagno et al. 2006). Also, a few lower mass YSOs have formed in the collected layer, for example in the direction of $\mathrm{C} 2$.

In order to compare with the predictions of the collect and collapse model of Whitworth et al. (1994), we need to know three parameters: 1) the Lyman continuum photon flux: we adopt $10^{49}$ ionizing photons per second, corresponding to the O5.5V-O6V exciting star (Martins et al. 2005); 2) the velocity dispersion in the collected layer: we measure a FWHM $1 \mathrm{~km} \mathrm{~s}^{-1}$ at the condensation peaks (Table 5), corresponding to a velocity dispersion $\sim 0.4 \mathrm{~km} \mathrm{~s}^{-1}$. The condensations are possibly collapsing, and thus a lower value, in the range 0.2 $0.3 \mathrm{~km} \mathrm{~s}^{-1}$, seems reasonable for the collected layer before collapse; 3) the density of the neutral material into which the H II region evolved: a density $\sim 500$ atom $\mathrm{cm}^{-3}$ allows formation of a 

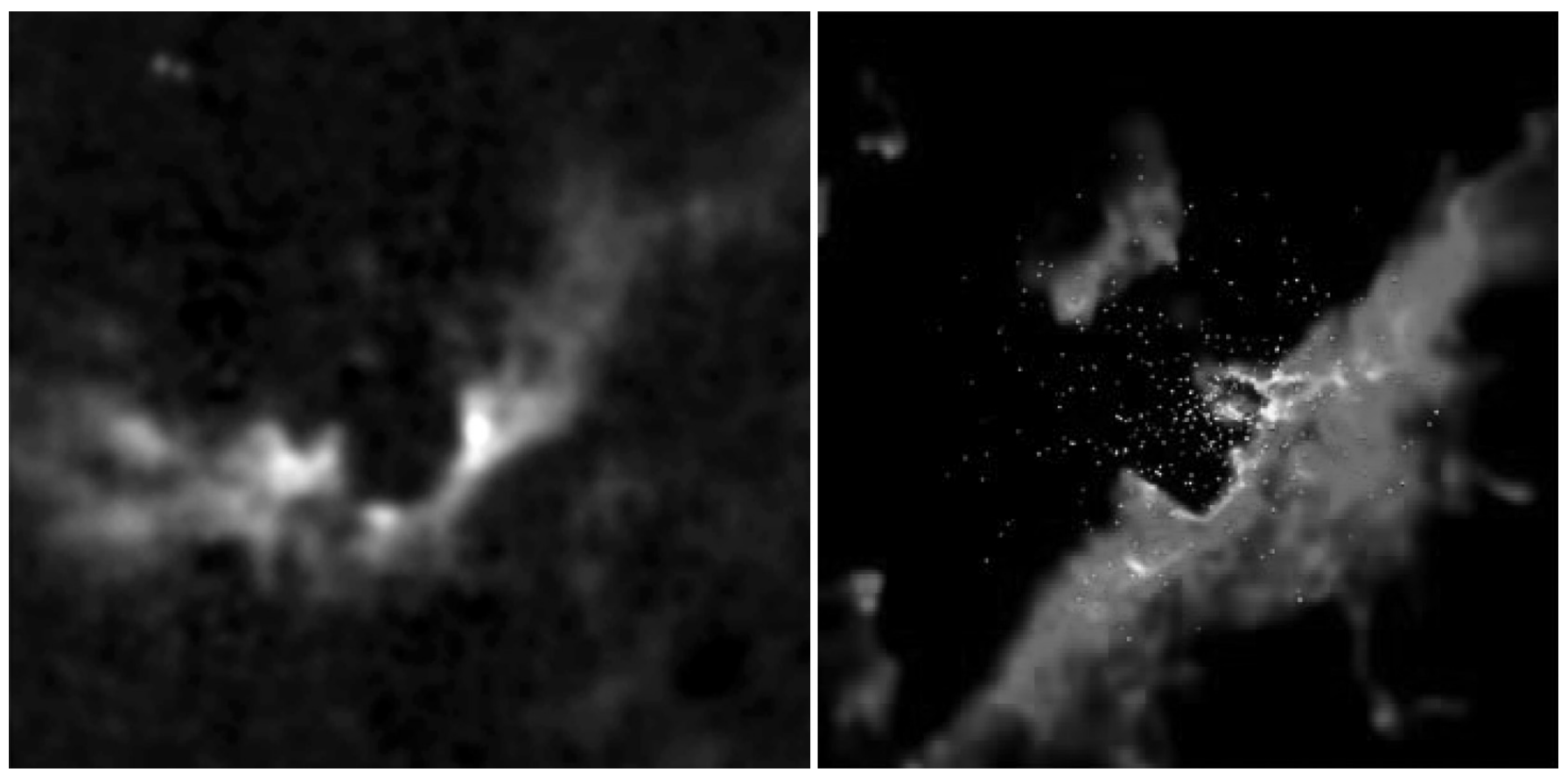

Fig. 14. Confrontation of observations and simulations. Left: ${ }^{12} \mathrm{CO}$ emission, integrated over the velocity. Right: simulation of a turbulent cloud illuminated by the UV radiation of a massive star (Dale et al. 2005, their Fig. 16).

collected layer (half a shell, of internal radius $2 \mathrm{pc}$ ) with a mass of about $750 M_{\odot}$, as observed. With these figures the model predicts the fragmentation of the collected layer after 2.2-2.8 Myr. It predicts the formation of fragments with a mass in the range 30-140 $M_{\odot}$, in agreement with the observations, separated by some 1.1-2.2 pc, again in agreement with the observations. But the radius of the $\mathrm{HII}$ region at the time of the fragmentation should be in the range $8.5-10.0 \mathrm{pc}$, much larger than the present radius of $2 \mathrm{pc}$. The fact that the $\mathrm{H}$ II region shows a champagne flow (and thus did not evolve in a homogeneous medium) probably explains why this model does not account for the observations. The possible presence of a magnetic field is an additional difficulty because, as demonstrated by Krumholz et al. (2006), it results in a non-spherical expansion.

The star formation efficiency can be estimated. Condensation 1 has a mass $\sim 220 M_{\odot}$ and has formed a (possibly isolated) B1V star; assuming for this star a mass of $14 M_{\odot}$, we obtain a star formation efficiency of $5 \%$.

\section{Conclusions}

The optical Galactic H II region Sh2-212 appears in the visible as a spherical H II region around its $05.5 \mathrm{~V}$ exciting star. The near-IR observations show that a rich cluster lies at its centre. A bright stellar object, No. 228, presenting a near-IR excess, and associated with a reflection nebulosity, lies on the border of Sh2212 , behind a bright rim. The MSX image at $8.3 \mu \mathrm{m}$ shows a bright point source in the direction of object No. 228, and radio continuum observations show the presence of a UC HII region in this exact direction. Sh2-212 lies in the middle of a molecular filament. Millimetre observations show that a thin molecular half-ring structure surrounds the brightest part of Sh2-212 at its back, and is expanding. This molecular layer is fragmented. The most massive fragment $\left(\sim 200 M_{\odot}\right)$ is associated with object No. 228. The SED of object No. 228 shows that it is a massive YSO of about $14 M_{\odot}$, hence able to ionize the UC H II region.
Low-density atomic hydrogen is detected at the periphery of the low-density ionized region.

We have tried to understand the star formation history in this region. Sh2-212 first formed and evolved inside a molecular filament. During its expansion neutral material was collected between the IF and the SF, as predicted by the collect and collapse process. This layer was then fragmented, and a secondgeneration massive star (No. 228) formed inside a massive fragment, ultimately ionizing a second-generation H II region. In a next stage, the IF bounding the Sh2-212 H II region reached the limits of the molecular filament, and the ionized region opened towards the low-density inter-condensation gas, creating a champagne flow. Presently, the Sh2-212 H II region is surrounded on one side by the dense collected molecular material, and on the other side by low-density atomic material.

The Sh2-212 H II region is, after Sh2-104 and RCW 79, one more example of massive-star formation by the collect and collapse process. It is a very special region for the following reasons:

- The massive YSO, No. 228, seems to have formed in isolation, or in a very small group. If this is confirmed, No. 228 is a very uncommon object.

- The layer of collected material is very well defined (circular, bright, thin, expanding); the fact that it survives in an inhomogeneous medium is especially interesting. This demonstrates that the collect and collapse process can work in a non-homogeneous medium, possibly in a turbulent one.

Acknowledgements. We gratefully thank D. Gravallon and S. Ilovaisky for the $\mathrm{H} \alpha$ and $\left[\mathrm{S}_{\mathrm{II}}\right]$ frames they obtained for us at the 120-cm telescope of the Observatoire de Haute-Provence, and M. Walmsley for constructive comments and questions. This work has made use of Aladin and of the Simbad astronomical database operated at CDS, Strasbourg, France. We have used data products from the Midcourse Space EXperiment and from the Two Micron All Sky Survey, obtained through the NASA/IPAC Infrared Science Archives. S.K. thanks the Laboratoire d'Astrophysique de Marseille and the Université de Provence for hosting him while some of this work was done. 


\section{References}

Blitz, L., Fich, M., \& Stark, A. A. 1982, ApJS, 49, 183

Brand, J., \& Blitz, L. 1993, ApJS, 49, 183

Beech, M., \& Mitalas, R. 1994, ApJS, 95, 517

Bernasconi, P. A., \& Maeder, A. 1996, A\&A, 307, 829

Caplan, J., Deharveng, L., Peña, M., et al. 2000, MNRAS, 311, 317

Chini, R., \& Wink, J. E. 1984, A\&A, 139, L5

Dale, J. E., Bonnell, I. A., Clarke, C. J., \& Bate, M. R. 2005, MNRAS, 358, 291

Deharveng, L., Peña, M., Caplan, J., \& Costero, R. 2000, MNRAS, 311, 329

Deharveng, L., Lefloch, B., Zavagno, A., et al. 2003, A\&A, 408, L25

Deharveng, L., Zavagno, A., \& Caplan, J. 2005, A\&A, 433, 565

Deharveng, L., Lefloch, B., Massi, F., et al. 2006, A\&A, 458, 191

Dyson, J. E., \& Williams, D. A. 1997, The physics of the interstellar medium, 2nd, ed. R. J. Tayler, \& M. Elvis (Bristol and Philadelphia: Institute of Physics Publishing)

Ellingsen, S. P. 2006, ApJ, 638, 241

Elmegreen, B. G. 1998, in ASP Conf. Ser. 148, ed. C.E. Woodward, J. M. Shull, \& H. A. Tronson, 150

Elmegreen, B. G., \& Lada, C. J. 1977, ApJ 214, 725

Fich, M. 1993, ApJS, 86, 475

Fich, L., Dahl, G., \& Treffers, R. 1990, AJ, 99, 622

Greve, A., Kramer, C., \& Wild, W. 1998, A\&AS, 133, 271

Hosokawa, T., \& Inutsuka, S. 2006, ApJ, 646, 240

Indebetouw, R., Mathis, J. S., Babler, B. L., et al. 2005, ApJ, 619, 931

Krumholz, M. R., Stone, J. M., \& Gardiner, T. A. 2006 [arXiv:astro-ph/0606539v2]
Lada, C. J., \& Adams, F. C. 1992, ApJ, 393, 278

Lockman, F. J. 1989, ApJS, 71, 469

Moffat, A. F. J., Ftzgerald, M. P., \& Jackson, P. D. 1979, A\&AS, 38, 197

Martins, F., \& Plez, B. 2006, A\&A, 457, 637

Martins, F., Schaerer, D., \& Hillier, D. J. 2005, A\&A, 436, 1049

Pişmiş, P., Hasse, I., \& Quintero, A. 1991, PASP, 103, 843

Price, S. D., Egan, M. P, Carey, S. J., Mizuno, D. R., \& Kuchar, T. A. 2001, AJ, 121,2819

Rieke, G. H., \& Lebofsky, M. J. 1985, ApJ, 288, 618

Roger, R. S., \& Leahy, D. A. 1993, AJ, 106, 31

Robitaille, T. P., Whitney, B. A., Indebetouw, R., \& Wood, K. 2007, ApJS 169, 328

Schmidt-Kaler, T. 1982, in Landolt-Börstein (New Series) Group VI/2, ed. K. Schaifers, \& H. H. Voigt, (Berlin: Springer-Verlag), 1

Schuster K.-F., Boucher, C., Brunswig, W., et al. 2004, A\&A, 423, 1171 Sharpless, S. 1959, ApJS, 4, 257

Shepherd, D. S., \& Churchwell, E. 1996, ApJ, 457, 267

Skrutskie, M. F., Cutri, R. M., Stiening, R., et al. 2006, AJ, 131, 1163

Smith, L. J., Norris, R. P. F., \& Crowther, P. A. 2002, MNRAS, 337, 1309

Stetson, P. B. 1987, PASP, 99, 191

Tenorio-Tagle, G. 1979, A\&A, 71, 59

Tokunaga, A. T. 2000, Allen's Astrophysical Quantities, ed. A. N. Cox, 4th Ed., (New York: Springer-Verlag), 143

Walmsley, M. 1995, RMAA, Ser. de Conf 1, 137

Whitworth, A. P., Bhattal, A. S., Chapman, S. J., Disney, M. J., \& Turner, J. A. 1994, MNRAS, 268, 291

Zavagno, A., Deharveng, L., Comerón, F., et al. 2006, A\&A, 171, 184 